\title{
Mineração
}

\section{Utilização do resíduo de caulim em blocos de vedação}

\section{(Use of kaolin waste for the production of cement bricks)}

\author{
Maria Luiza de S. Rezende \\ Mestranda, COPEAG, UFCG.E-mail:mlresen@yahoo.com.br \\ Romualdo R. Menezes \\ Pesquisador, Dr., Unidade Acadêmica de Eng. de Materiais, UFCG.E-mail:romualdomenezes@dema.ufcg.edu.br \\ Gelmires de Araújo Neves \\ Prof., Dr., Unidade Acadêmica de Eng.de Materiais, UFCG.E-mail: gelmires@dema.ufcg.edu.br \\ José Wallace B. Nascimento \\ Prof., Dr., Unidade Acadêmica de Eng. Agrícola, UFCG. E-mail: wallace@deag.ufcg.edu.br \\ Antonio Farias Leal \\ Prof., Dr., Unidade Acadêmica de Eng. Agrícola, UFCG. E-mail: leal@deag.ufcg.edu.br
}

\section{Resumo}

O caulim é uma argila com um grande número de aplicações industriais, no entanto sua produção gera uma enorme quantidade de resíduos. Assim, esse trabalho tem, por objetivo, estimar o volume de resíduo da produção de caulim acumulado na região produtora do Estado da Paraíba e analisar sua viabilidade como matéria-prima alternativa para a produção de blocos de alvenaria de vedação. O resíduo da produção do caulim foi classificado segundo sua periculosidade ambiental e, em seguida, caracterizado através da determinação de sua composição química e características físicas. A quantidade de resíduo acumulado foi estimada com base em imagens de satélite. Em seguida, blocos de vedação cimento-areia foram preparados substituindo parte da areia pelo resíduo. Determinaram-se a resistência à compressão simples dos blocos de vedação e a sua absorção de água. Com base nos resultados, pode-se concluir que o resíduo de caulim é classificado como não perigoso e não inerte, que o volume estimado de resíduo supera 2,6 milhões de toneladas e que os blocos confeccionados com o resíduo, com substituição em até $70 \%$ de areia, são adequados para alvenaria de vedação.

Palavras-chave: Reciclagem, resíduo de caulim, blocos de vedação.

\begin{abstract}
Kaolin is a clay used in several industrial applications, however, its production generates large amount of waste. Thus, the aim of this work is estimate the amount of kaolin production waste accumulated in the production region of the Paraiba state,Brazil, and evaluate its suitability as an alternative raw material for the production of masonry blocks without structural function. The waste was classified according to environmental laws, and characterized by chemical composition and physical characteristics determination. Satellite images were used to estimate the amount of accumulated waste. Cement-sand masonry blocks were prepared partially substituting sand by kaolin waste and their compression strength and water absorption were determined. The waste was classified as nondangerous but non-inert material, and the estimated amount of accumulated waste was higher than 2.6 millions tons. Sand can be partially substituted for kaolin waste in amounts of up to $70 \%$ in the production of masonry blocks.
\end{abstract}

Keywords: Recycling, kaolin waste, masonry block. 


\section{Introdução}

O caulim é uma das argilas industriais mais utilizadas mundialmente, em virtude de suas excelentes propriedades naturais, tais como: brancura, granulometria muito fina das partículas, pequena abrasão e grande inércia ou estabilidade química (Moura, 1981). A primeira aplicação industrial do caulim foi na fabricação de artigos cerâmicos, entretanto, atualmente, o caulim é utilizado nos mais diversos setores industriais, como o de papel, borracha, plásticos, pesticidas, rações, produtos alimentícios e farmacêuticos e fertilizantes (DNPM, 2001).

A extração e o beneficiamento do caulim produzem uma enorme quantidade de resíduos, estimada em torno de 80 a $90 \%$ do volume bruto explotado. Esses resíduos são, em geral, descartados indiscriminadamente em campo aberto, desprezando-se as exigências de utilização de aterros e provocando uma série de danos ao meio ambiente e à saúde da população residente nas regiões circunvizinhas aos "depósitos" de resíduos.

Segundo o CONAMA (2005), impacto ambiental pode ser definido como qualquer alteração nas características físicas, químicas e/ou naturais do meio ambiente, causada por qualquer forma de matéria ou energia resultante de atividades humanas, que, direta ou indiretamente, afetem: a saúde, segurança e bemestar da população; as atividades sociais e econômicas; o conjunto de plantas e animais de uma determinada área; as condições estéticas e sanitárias do meio ambiente e a qualidade dos recursos naturais. Assim, observa-se que o resíduo do processamento do caulim provoca uma série de impactos ambientais ao meio físico e biótico.

No Estado da Paraíba, há um agravante ao descarte indiscriminado dos resíduos de caulim, que é o seu acúmulo em torno das empresas de mineração há dezenas de anos, o que vem sendo alvo de severas fiscalizações e tem preocupado ambientalistas e pesquisadores, por não ser possível precisar os efeitos futuros das montanhas de resíduos na flora e fauna da região. O próprio mon- tante de resíduos existente na região não é precisamente estimado, o que faz com que a imprecisão dobre e que os impactos futuros desses materiais se intensifiquem ainda mais.

O acúmulo de resíduos no beneficiamento do caulim vem se tornando motivo de estudos por diversos pesquisadores (Silva et alii., 2001, Menezes et alii., 2007a, 2007b, 2007c, Rezende, 2007), buscando-se minimizar ou, até mesmo, solucionar os danos ambientais provocados por esses materiais, quer seja pela otimização do método de explotação e armazenamento ou pela apresentação de alternativas de reutilização do resíduo como matéria-prima alternativa em diversos setores.

No entanto, para que haja uma possível utilização racional do resíduo, na tentativa de minimizar os seus impactos ambientais, é necessário que seja feito um levantamento, tanto da quantidade de resíduos já existentes e gerados nos pólos de beneficiamento, quanto das características físicas e mineralógicas desses materiais.

Com base em dados da quantidade disponível e das características do resíduo, é possível o seu direcionamento para o ciclo produtivo, a fim de utilizar parte do volume de resíduo a ser descartado e economizar matérias-primas não renováveis e energia, de modo a contribuir para o desenvolvimento sustentável. Assim, esse trabalho teve por objetivo estimar o volume do resíduo da produção de caulim depositada na região produtora do Estado da Paraíba e analisar sua viabilidade como matéria-prima alternativa para a produção de blocos de vedação.

\section{Materiais e métodos}

Nessa pesquisa, foram utilizados os seguintes materiais: resíduo da produção do caulim, coletado, conforme a NBR 10007 (ABNT, 2004a) na empresa Caulisa - Comércio e Beneficiamento de Caulim Ltda., localizada no município de Juazeirinho, PB; cimento portland tipo CPII Z-32, marca ZEBU (Cimpor Cimen- tos do Brasil, João Pessoa, PB); areia do rio Paraíba extraída do município de Barra de Santana, PB, com diâmetro máximo de $4,8 \mathrm{~mm}$ e teor de pulverulentos de $0,71 \%$. O resíduo da produção do caulim foi caracterizado em trabalhos anteriores (Lima, 2005), sendo observado que é constituído por mica, caulinita e quartzo.

$\mathrm{O}$ resíduo foi passado em peneira com abertura de $9,5 \mathrm{~mm}$ e classificado quanto ao risco ambiental, segundo a NBR 10004 (ABNT, 2004b), através de ensaios de lixiviação, NBR 10005 (ABNT, 2004c), e solubilização, NBR 10006 (ABNT, 2004d) (os extratos foram analisados no Laboratório de Física e Química do Solo do CCA, UFPB). Em seguida, o resíduo foi caracterizado através da determinação da massa unitária em estado solto (MUES), NBR 7251 (ABNT, 1982), teor de materiais pulverulentos (TMP), NBR 7219 (ABNT 1983a) e distribuição granulométrica, NBR 7217 (ABNT, 1987). A composição química do resíduo foi determinada no Laboratório de Análises Minerais do CCT, UFCG.

A estimativa do volume de resíduo acumulado na região produtora do Estado da Paraíba foi realizada com base em imagens de satélite TM/LANDSAT-5, realizadas em 2001, fornecidas pelo Laboratório de Sensoriamento Remoto e SIG do CCT/ UFCG. A altura média dos volumes de resíduos foi considerada como $1,80 \mathrm{~m}$, com base em observações nos locais de depósitos. A estimativa dos volumes presentes em 2001 foi atualizada, considerando-se a produção mensal de resíduos fornecida pelo Cadastro Industrial da Superintendência de Administração do Meio Ambiente da Paraíba (SUDEMA).

Após a caracterização e estimativa do volume, foram moldados blocos com dimensões de $90 \mathrm{~mm}$ x $190 \mathrm{~mm}$ x $390 \mathrm{~mm}$ com cimento e areia na proporção de 1:6 (traço 1:6). A areia foi substituída pelo resíduo de caulim nas proporções de 40, 70 e $100 \%$. Em seguida, os corpos-deprova foram curados por 7,28 e 63 dias e determinadas a sua resistência à compressão simples, segundo a NBR 7171 
Maria Luiza de S. Rezende et al.

(ABNT 1992), e a sua absorção de água após 28 dias de cura.

\section{Resultados e discussão}

Na Tabela 1, estão apresentados os valores da análise do extrato lixiviado. Com base nos resultados, verifica-se que o resíduo não apresenta características de toxidade. Como o resíduo não apresenta características de inflamabilidade, corrosividade, reatividade ou patogenicidade, é considerado não perigoso, classe II, segundo a NBR 10004 (ABNT, 2004b).

$\mathrm{Na}$ Tabela 2, estão apresentados os valores da análise do extrato solubilizado. Com base nos resultados, observase que o extrato apresenta concentra- ções de Cd e Mn acima dos limites definidos na NBR 10004 (ABNT, 2004b), o que mostra que o resíduo, apesar de não tóxico, é não inerte, sendo classificado como classe IIA, segundo a NBR 10004 (ABNT, 2004b). Essa classificação evidencia que o resíduo necessita ser descartado em aterros sanitários, o que não ocorre na prática e vem ao encontro das preocupações com o grande acúmulo desse resíduo a céu aberto há dezenas de anos.

Na Tabela 3, estão apresentados os valores da distribuição granulométrica e, na Tabela 4, os resultados da caracterização física do resíduo de caulim. Comparando os resultados com os especificados para agregado miúdo, NBR 7211 (ABNT, 1983b), observa-se que o resíduo apresentou valor de massa unitária inferior e teor de material pulverulento superior ao indicado na normalização. Em relação à distribuição granulométrica, verifica-se que o diâmetro máximo foi de $9,5 \mathrm{~mm}$ e o teor de pulverulentos de $2,83 \%$.

A composição química do resíduo está contida na Tabela 5. Observa-se que o resíduo apresenta um elevado teor de $\mathrm{SiO}_{2}$, o que, juntamente com o teor de $\mathrm{Al}_{2} \mathrm{O}_{3}$ e com os dados de caracterização obtidos anteriormente (Lima, 2005), indica que o resíduo é constituído, predominantemente, por quartzo (areia), indicando que, provavelmente, pode ser utilizado em argamassas em substituição de parte do agregado miúdo.

Na Figura 1, está presente uma das imagens utilizadas para a estimativa do volume de resíduos depositado na região produtora de caulim do Estado da

Tabela 1 - Resultado da análise do extrato lixiviado.

\begin{tabular}{c|c|c|c|c|c|c|c|c|c|c|c}
\hline & $\mathbf{C d}$ & $\mathrm{Cl}^{-}$ & $\mathbf{N a}$ & $\mathbf{C u}$ & $\mathbf{F e}$ & $\mathbf{M n}$ & $\mathbf{Z n}$ & $\mathbf{P b}$ & $\mathbf{C r}$ & $\mathbf{A l}$ & $\mathbf{S O}^{4}$ \\
\hline Extrato (mg/L) & 0,07 & 339,5 & 1.003 & 0,1 & 0,01 & 0,24 & $<0,01$ & 0,58 & 0,05 & 755,44 & 11,22 \\
\hline Limite $^{\mathrm{a}}$ (mg/L) & 0,5 & $\mathrm{NE}^{\mathrm{b}}$ & $\mathrm{NE}^{\mathrm{b}}$ & $\mathrm{NE}^{\mathrm{b}}$ & $\mathrm{NE}^{\mathrm{b}}$ & $\mathrm{NE}^{\mathrm{b}}$ & $\mathrm{NE}^{\mathrm{b}}$ & 1,0 & 5,0 & $\mathrm{NE}^{\mathrm{b}}$ & $\mathrm{NE}^{\mathrm{b}}$ \\
\hline
\end{tabular}

${ }^{a}$ ABNT 2004b. $\quad{ }^{\text {b }}$ Não especificado.

Tabela 2 - Resultado da análise do extrato solubilizado.

\begin{tabular}{c|c|c|c|c|c|c|c|c|c|c|c}
\hline & $\mathbf{C d}$ & $\mathbf{C l}^{-}$ & $\mathbf{N a}$ & $\mathbf{C u}$ & $\mathbf{F e}$ & $\mathbf{M n}$ & $\mathbf{Z n}$ & $\mathbf{P b}$ & $\mathbf{C r}$ & $\mathbf{A l}$ & $\mathbf{S O}^{\mathbf{4}}$ \\
\hline Extrato (mg/L) & 0,035 & 17,5 & 10,5 & $<0,01$ & $<0,01$ & 0,35 & $<0,01$ & 0,01 & 0,05 & 0 & 14,29 \\
\hline Limite $^{\mathbf{a}}$ (mg/L) & 0,005 & 250,0 & 200,0 & 2,0 & 0,3 & 0,10 & 5,0 & 0,01 & 0,05 & 0,2 & 250,0 \\
\hline
\end{tabular}

${ }^{\text {a }}$ ABNT 2004b.

Tabela 3 - Distribuição granulométrica do resíduo de caulim.

\begin{tabular}{c|c|c|c|c|c|c|c|c|c}
\hline Diâmetro $(\mathbf{m m})$ & 9,5 & 6,3 & 4,8 & 2,4 & 1,2 & 0,6 & 0,3 & 0,15 & Resto \\
\hline (\%) retida & 1,14 & 2,49 & 4,72 & 21,53 & 48,82 & 73,17 & 88,54 & 97,17 & 100 \\
\hline
\end{tabular}

Tabela 4 - Características físicas do resíduo de caulim.

\begin{tabular}{c|c|c|c}
\hline Características & MUES $^{\mathbf{a}}\left(\mathbf{k g} / \mathbf{m}^{\mathbf{3}}\right)$ & Dimensão Máxima (mm) & Teor de Pulverulentos (\%) \\
\hline Resultado & 1280 & 9,5 & 2,83 \\
\hline
\end{tabular}

a Massa unitária específica solta. 
Tabela 5 - Composição química (\%) do resíduo de caulim.

\begin{tabular}{c|c|c|c|c|c|c|c}
\hline $\mathbf{S i O}_{2}$ & $\mathbf{A l}_{2} \mathbf{O}_{3}$ & $\mathbf{F e}_{2} \mathbf{O}_{3}$ & $\mathbf{C a O}$ & $\mathbf{M g O}$ & $\mathbf{N a}_{\mathbf{2}} \mathbf{O}$ & $\mathbf{K}_{\mathbf{2}} \mathbf{O}$ & $\mathbf{P F}^{\mathbf{a}}$ \\
\hline 77,56 & 16,09 & 0,16 & Traços & Traços & 0,04 & 0,42 & 5,68 \\
\hline
\end{tabular}

${ }^{a}$ PF - Perda ao fogo.

Paraíba. Com base nas imagens de satélite, foram calculadas as áreas dos depósitos e, utilizando a massa unitária em estado solto (Tabela 4) e as observações realizadas em campo das alturas dos depósitos, em torno de $1,8 \mathrm{~m}$, foram calculadas as quantidades de resíduos presentes nos depósitos.

Na Tabela 6, estão apresentadas as quantidades acumuladas de resíduo em cada município estudado (região produtora do Estado). Observa-se que a estimativa conduz a um volume acumulado em 2001 superior a dois milhões de toneladas de resíduo (2.116.637 ton). Entretanto a produção média de resíduos por ano desde de 2001 é de 6.800 toneladas/ mês segundo a SUDEMA (SUDEMA, 2006), o que, somado ao montante já existente, totaliza uma estimativa de, aproximadamente, 2,6 milhões de toneladas de resíduos descartadas a céu aberto atualmente.

A estimativa presente na Tabela 6 foi efetuada considerando-se que não há remoção de estéreis e preenchimento de cavas abandonadas por parte dos mineradores, o que diminuiria a quantidade de resíduo acumulado, fazendo dos resultados obtidos uma superestimativa.

Vale salientar que o procedimento de preenchimento de cavas poderia minimizar o impacto ambiental da extração e beneficiamento do caulim, entretanto não é efetuado pelos mineradores em nenhuma dos municípios produtores paraibanos, o que está associado à criação da reserva garimpeira nas regiões produtores de caulim em 1982. O intuito da criação da reserva foi evitar conflitos entre mineradores e garimpeiros, proibindo a lavra mecanizada. No entanto, o garimpeiro, como não beneficia o minério, se exime da responsabilidade com o resíduo gerado no beneficiamento, en-

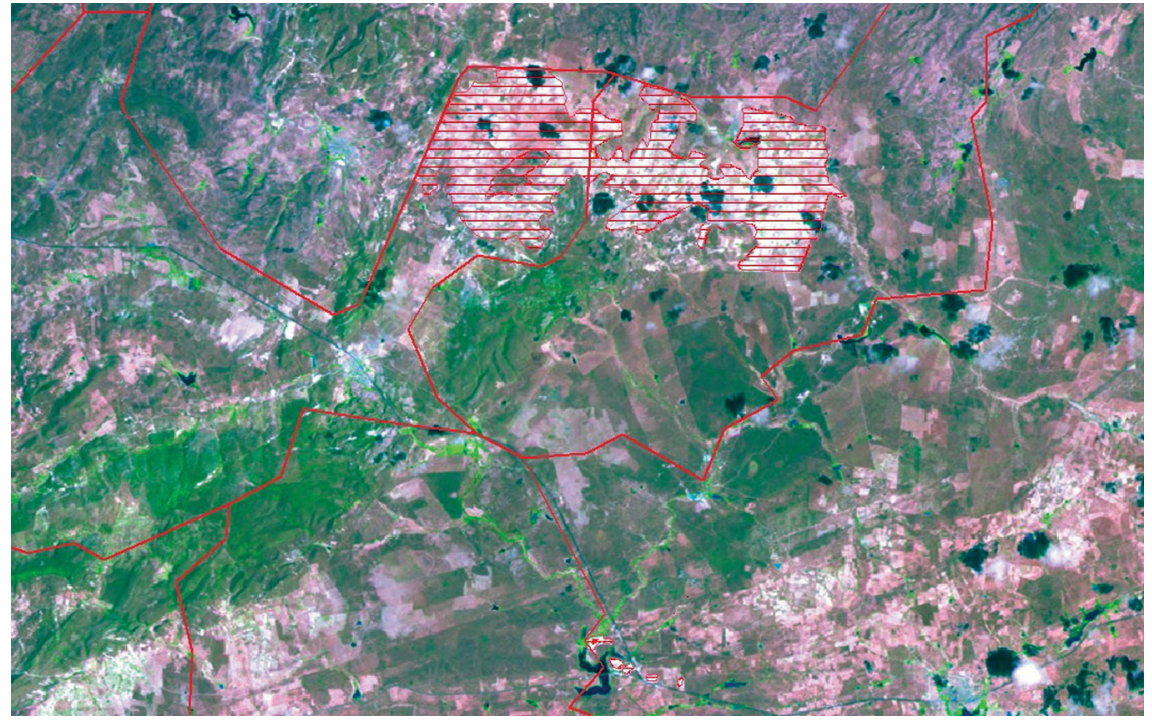

Figura 1 - Imagens de depósitos de rejeitos de caulim nos municípios de Tenório e Junco do Seridó (linhas vermelhas representam limites municipais e as áreas tracejadas representam os depósitos de resíduo de caulim).

Tabela 6 - Estimativa de volume acumulado de resíduo de caulim na região produtora do Estado da Paraíba.

\begin{tabular}{c|c|c|c}
\hline Município & Área $\left(\mathbf{m}^{2}\right)$ & Volume $\left(\mathbf{m}^{\mathbf{3}}\right)$ & Massa $(\mathbf{k g})$ \\
\hline Assunção & 128.260 & 280.860 & 359.500 .800 \\
\hline Juazeirinho & 172.646 & 310.763 & 397.776 .640 \\
\hline Junco do Seridó & 370.000 & 666.000 & 852.480 .000 \\
\hline Tenório & 220.000 & 396.000 & 506.880 .000 \\
\hline
\end{tabular}

quanto as empresas de beneficiamento ficam impossibilitadas de remover o resíduo para as cavas por não ter acesso às mesmas.

A grande quantidade de resíduo disponível nos depósitos em campo aberto destaca dois pontos muito importantes para o seu gerenciamento nos próximos anos. Primeiro, é necessário que seja dada destinação urgente ao resíduo gerado, já que, por se tratar de um resíduo IIA, pode provocar contaminações nas reservas de água da região semi-árida onde está inserido. Segundo, alterna- tivas de reciclagem tecnologicamente eficientes não terão a adversidade, por vezes determinante no seu sucesso econômico, de disponibilidade de matériasprimas, já que o volume existente pode suprir várias empresas de pequeno porte, destinadas à confecção de blocos para alvenaria de fechamento.

Na Figura 2, estão apresentados os resultados de resistência à compressão simples e fator água/cimento, $\mathrm{f}_{\mathrm{a} / \mathrm{c}}$, das composições estudadas. Pode-se observar que o fator água/cimento aumentou com o teor de resíduo utilizado, o que 
está associado ao maior teor de finos do resíduo em relação ao agregado miúdo. Verifica-se que os valores de comportamento mecânico dos blocos contendo resíduo foram inferiores aos do convencional. Entretanto, comparando-se os resultados obtidos com os especificados para blocos de vedação, NBR 7171 (ABNT, 1992) (valores acima de $1 \mathrm{MPa}$ ), observa-se que os blocos com 40 e $70 \%$ de resíduo apresentam valores dentro das especificações. Após os 63 dias de cura, todos os blocos de alvenarias apresentaram valores de resistência superiores aos requisitados, sendo assim, com esses resultados, espera-se que os blocos em estudo possam ser utilizados na construção de habitações populares e instalações agrícolas nas cidades circunvizinhas dos locais geradores deste resíduo.

$\mathrm{Na}$ Tabela 7, estão apresentados os valores de absorção de água após 28 dias de cura. Pode-se observar que a utilização do resíduo provoca um leve aumento na absorção de água dos blocos. No entanto, todos os valores estão de acordo com a faixa de absorção (8 a 25\%) especificada pela NBR 7171 (ABNT, 1992). Esse aumento na absorção pode estar associado ao menor empacotamento do sistema com a utilização do resíduo, apesar de não ocorrer grandes alterações da absorção com o aumento do teor de resíduo de 40 para $70 \%$.

\section{Conclusões}

Esse trabalho teve por objetivo estimar o volume do resíduo da produção de caulim acumulado na região produtora do Estado da Paraíba e analisar sua viabilidade como matéria-prima alternativa para a produção de blocos de vedação. Com base nos resultados obtidos, pode-se concluir que:

- O resíduo da produção do caulim é classificado como não perigoso, não inerte, classe IIA, conforme a normalização brasileira.

- O resíduo possui características físicas similares ao do agregado miúdo

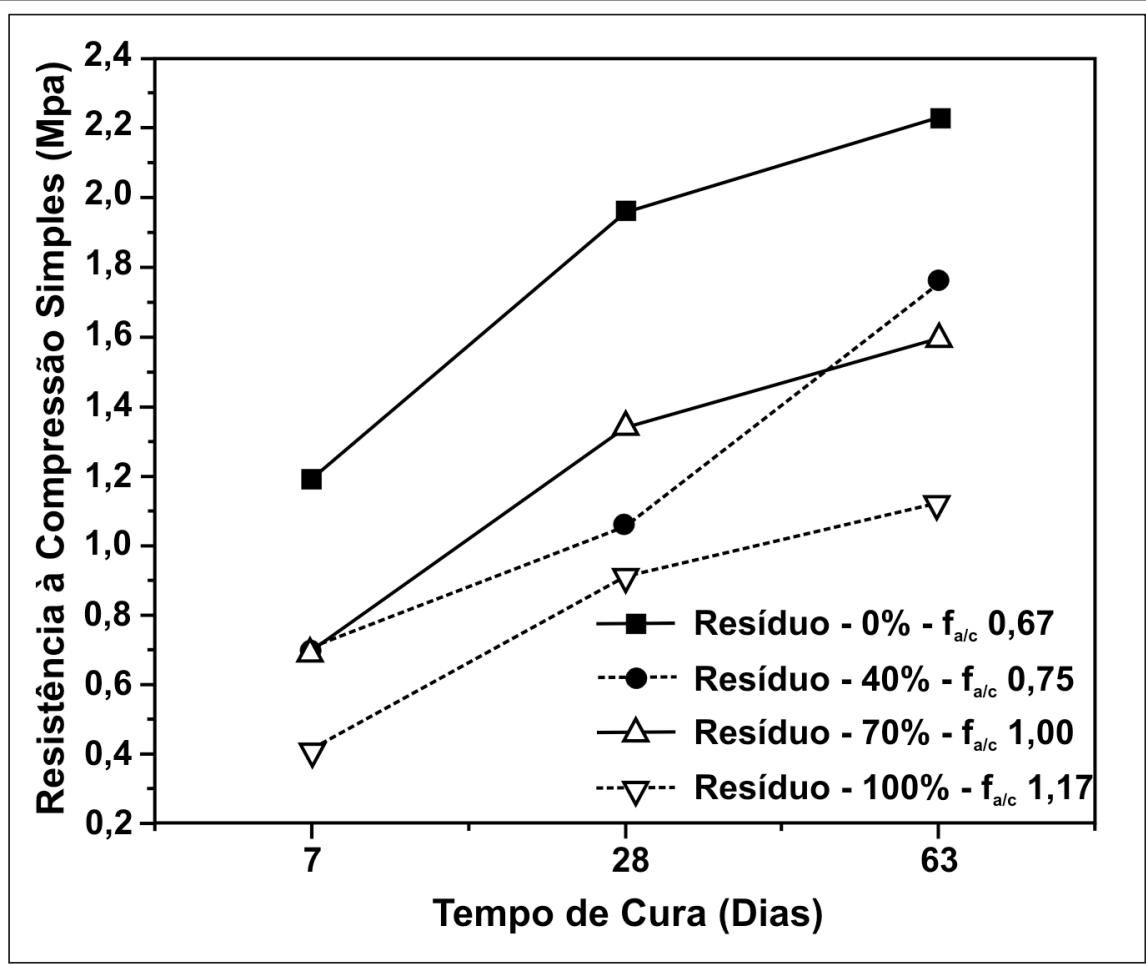

Figura 2 - Resistência à compressão simples das composições estudadas.

Tabela 7 - Absorção de água dos blocos após 28 dias de cura.

\begin{tabular}{c|c|c|c|c}
\hline Resíduo (\%) & 0 & 40 & 70 & 100 \\
\hline Absorção (\%) & 11,6 & 13,7 & 13,2 & 16,0 \\
\hline
\end{tabular}

utilizado na produção de blocos de vedação cimento-areia.

- O volume estimado de resíduo acumulado na região produtora do Estado da Paraíba está na ordem de 2,6 milhões de toneladas, constituindose em um grave problema ambiental.

- A utilização do resíduo, na produção de blocos de alvenaria de vedação cimento-areia em substituição parcial ao agregado miúdo em teores de até $70 \%$, constitui boa alternativa para diminuir o impacto ambiental, sendo uma aplicação com viabilidade econômica.

\section{Agradecimentos}

Os autores agradecem ao $\mathrm{CNPq}$ (471414/04-9) e à FAPESQ/MCT/CNPq (003/03 e 004/06) pelo apoio financeiro.

\section{Referências} bibliográficas

ABNT - ASSOCIAÇÃO BRASILEIRA DE NORMAS TÉCNICAS. NBR 7251: Agregado em estado solto - determinação da massa unitária. Rio de Janeiro: 1982.

ABNT - ASSOCIAÇÃO BRASILEIRA DE NORMAS TÉCNICAS. NBR 7219: Agregados - determinação do teor de materiais pulverulentos. Rio de Janeiro: 1983a.

ABNT - ASSOCIAÇÃO BRASILEIRA DE NORMAS TÉCNICAS. NBR 7211: Agregado para concreto-especificação. Rio de Janeiro: 1983b.

ABNT - ASSOCIAÇÃO BRASILEIRA DE NORMAS TÉCNICAS. NBR 7217: Agregados - determinação da composição granulométrica. Rio de Janeiro: 1987.

ABNT - ASSOCIAÇÃO BRASILEIRA DE NORMAS TÉCNICAS. NBR $7 \mathbf{1 7 1}$ : Bloco cerâmico para alvenaria. Rio de Janeiro: 1992. 
Utilização do resíduo de caulim em blocos de vedação

ABNT - ASSOCIAÇÃO BRASILEIRA DE NORMAS TÉCNICAS. NBR 10007: Amostragem de resíduos sólidos. Rio de Janeiro: 2004a.

ABNT - ASSOCIAÇÃO BRASILEIRA DE NORMAS TÉCNICAS. NBR 10004: Resíduos sólidos - classificação. Rio de Janeiro: 2004b.

ABNT - ASSOCIAÇÃO BRASILEIRA DE NORMAS TÉCNICAS. NBR 10005: Procedimento para obtenção de extrato lixiviado de resíduos sólidos. Rio de Janeiro: 2004c.

ABNT - ASSOCIAÇÃO BRASILEIRA DE NORMAS TÉCNICAS. NBR 10006: Procedimento para obtenção de extrato solubilizado de resíduos sólidos. Rio de Janeiro: 2004d.

CONAMA - CONSELHO NACIONAL DE MEIO AMBIENTE. Resolução $\boldsymbol{n}^{\boldsymbol{o}}$. 1524/1982. Disponível em <http:// $\mathrm{w} \mathrm{w} \mathrm{w} \cdot \mathrm{d} \mathrm{n} \mathrm{p} \mathrm{m.g} \mathrm{o} \mathrm{v.} \mathrm{b} \mathrm{r} \mathrm{/}$ conteudo.asp? Idsecao $=67 £$ IDPagina $=84 £$ IDLegislacao $=60>$. Acesso em 20 out. 2005.
DNPM - DEPARTAMENTO NACIONAL DE PRODUÇÃO MINERAL. Anuário Mineral Brasileiro de 2001. Brasília: DNPM, 2001. v. 1.

LIMA, M. S. Utilização do resíduo de caulim para uso em blocos de concreto sem função estrutural. Campina Grande: Universidade Federal de Campina Grande, 2005. 74f. (Dissertação de Mestrado em Engenharia Civil).

MENEZES, R. R. et alii. Utilização do resíduo do beneficiamento do caulim na produção de blocos e telhas cerâmicos. Revista Matéria, v. 12, n. 1, p. 226-236, 2007a.

MENEZES, R. R. et alii. Análise da coutilização do resíduo do beneficiamento do caulim e serragem de granito para produção de blocos e telhas cerâmicos. Cerâmica, v. 53, p. 192-199, 2007 b.

MENEZES, R. R. et alii. Utilização do resíduo do beneficiamento do caulim para produção de corpos mulíticos. Cerâmica, v. 53, p. 388-395, 2007c.
MOURA, O. J. M. Programa Pegmatitos. Belo Horizonte: Governo do Estado de Minas Gerais/Metamig, 1981.

REZENDE, M. L. S. Estudo de viabilidade técnica da utilização do resíduo de caulim em blocos de vedação. Campina Grande: Coordenação de Pós-Graduação em Engenharia Agrícola, Universidade Federal de Campina Grande, 2007. 54 f. (Dissertação de Mestrado em Engenharia Agrícola).

SILVA, A. C., VIDAL, M., PEREIRA, M. G. Impactos ambientais causados pela mineração e beneficiamento de caulim. REM - Revista Escola de Minas, v. 54, n. 2, p. 133-136, 2001.

SUDEMA - SUPERINTENDÊNCIA DE ADMINISTRAÇÃO DO MEIO AMBIENTE. Cadastro Industrial. João Pessoa: SUDEMA, 2006.

\section{Artigo recebido em 20/08/2007 e aprovado em 07/05/2008.}

